\title{
Postoperative Complications and Oncologic Outcomes after Resection of Colorectal Liver Metastases: The Importance of Staying on Track
}

\author{
Ching-Wei D. Tzeng, MD and Jean-Nicolas Vauthey, MD \\ Department of Surgical Oncology, The University of Texas MD Anderson Cancer Center, Houston, TX
}

In a meta-analysis of 2,280 patients who underwent surgery for colorectal liver metastases (CLM) at four major hepatobiliary centers, Matsuda et al. ${ }^{1}$ review recent surgical series for which the association between postoperative and long-term outcomes was reported. Despite major advances in liver surgery in the past two decades, during which 30-day mortality decreased to $2.4 \%$ nationally for hepatectomies, the authors emphasize the continued high rate $(\sim 40-50 \%)$ of postoperative complications, which occur more frequently with operations of greater magnitude, or in association with comorbidities. ${ }^{2,3}$

Whether the association between complications and survival is the result of a direct cause-effect relationship or a reflection of more extensive surgery being performed for greater tumor burden (which would negatively affect survival) cannot be proven, but the merit of this analysis is that it brings to the forefront the need for "staying on track" in hepatobiliary oncology, i.e., making sure that patients complete the recommended multiple stages of treatment. This is particularly relevant in cancer patients who need multidisciplinary therapy because veering off track will delay additional treatment, whether it is chemotherapy, radiation therapy, or a planned second-stage operation. ${ }^{4-7}$ As such, the return to intended oncologic therapy (any form) and, similarly, the return to full preoperative performance status are major endpoints that are currently underreported in most outcome studies on surgical morbidity. So, if we accept the conclusions of the authors, how do we "stay on track" in order to decrease complications in the surgical treatment of CLM?

The first modifiable risk factor is the duration of preoperative chemotherapy, which is increasingly used for

(C) Society of Surgical Oncology 2013

Published Online: 12 April 2013

J.-N. Vauthey, MD

e-mail: jvauthey@mdanderson.org
CLM. Chemotherapy for an extended duration ( $>8$ cycles) only increases the risk of chemotherapy-associated liver injury and postoperative complications without improving pathologic response. ${ }^{8}$ Thus, every patient with CLM who might be a candidate for surgery (this includes patients with "initially unresectable" CLM who have bilateral disease, small livers, or resectable extrahepatic disease) should be evaluated at diagnosis by a multidisciplinary team that includes a hepatobiliary surgeon. The goal of chemotherapy is not and should not be complete disappearance of the CLM. Effective first-line chemotherapy should be used, because multiple lines of chemotherapy are associated with inferior survival. ${ }^{9}$

A second method of decreasing morbidity is the sequencing of operations in the setting of synchronous CLM with intact primary colorectal cancer. The liver-first, or reverse, approach allows the less contaminated operation to be done before resection of the primary while clearing the patient of metastatic disease. As an option for patients with nonobstructing primary tumors, this is usually chosen for patients requiring neoadjuvant chemotherapy and larger hepatic resections for CLM that are the dominant lifelimiting process (rather than the primary lesion) for the patient. ${ }^{4,5}$ The reverse approach should be considered in the subset of patients who require major (right) liver resection with the primary in place, because the mortality and morbidity of combined resection of liver and primary is markedly increased in these patients. ${ }^{10}$ Concerns about the morbidity of major colorectal operations, even proctectomies, staged after major hepatectomies should not preclude reverse-approach sequencing. ${ }^{11}$

Third, dividing extended hepatectomies into two stages continues to be a viable option in patients with bilateral CLM. ${ }^{6}$ Staged resection is recommended for any of the following situations: inadequate future liver remnant (FLR), medical comorbidities precluding longer operative time for simultaneous resection of the CLM and primary 
tumor, and/or major operations needed at both sites (e.g., major/extended hepatectomy and proctectomy). It is important to keep the two-stage approach in the armament of the hepatobiliary surgeon. Often, careful sequencing is a better tool than fancier resections of excessive extent.

A fourth way to decrease morbidity is the use of portal vein embolization (PVE), either between stages of a twostage hepatectomy or before a major/extended one-stage hepatectomy. With CT volumetry so ubiquitous, the days of "eyeballing" a CT scan to estimate FLR should be over. Up to $75 \%$ of patients at presentation have insufficient FLR for an extended right hepatectomy based on segments II + III alone. PVE allows a functional stress test to see the regenerative capacity of the FLR. During a median 4-5 weeks between PVE and hepatectomy, surgeons study both the degree of hypertrophy ( $\mathrm{DH}$, needs to be $>5 \%$ ) and the kinetic growth rate (DH divided by time in weeks), a promising tool that may help us reach the goals of minimal hepatic insufficiency and $0 \%$ liver-related mortality. ${ }^{12}$ Patients with a kinetic growth rate of $<2 \%$ may be spared a complex hepatectomy that would have left an insufficient FLR for safe recovery. It also is important to point out that not all PVEs are equal. With aggressive PVE of the right portal vein and segment IV and the use of spherical microspheres and coils, an average FLR increase of $69 \%$ has been documented. $^{13}$

A fifth method to decrease morbidity in the majority of patients presenting with multiple metastases is the use of nonanatomic partial hepatectomies to achieve multiple R0 resections rather than sacrificing parenchyma with formal hemihepatectomies. ${ }^{14}$ With improved systemic therapy, this paradigm may be evolving, as margin-negative resections have become oncologically acceptable. ${ }^{15}$ In addition, in the new era of effective systemic therapy, we would consider microscopic margin-positive resection in patients who have an optimal morphologic response to chemotherapy. ${ }^{16,17}$ Recently, we have shown similar survival between patients who had $\mathrm{R} 0$ resections and those who had $\mathrm{R} 1$ resections with response to chemotherapy. ${ }^{18}$

The final question to bring forth is whether there is a direct association between complications and oncologic outcomes after resection of CLM. Are there greater complications with bigger operations for more extensive cancers? Are these more extensive tumor burdens a predictor of worse prognosis? Do the postoperative complications themselves directly affect the immune system or stimulate an inflammatory response that triggers growth-signaling cytokines? Or do they harm survival by compromising additional treatment? The meta-analysis of Matsuda et al. reports an association, and further studies will be needed to resolve the question of causation.

In conclusion, we commend Matsuda et al. for reviewing in a meta-analysis the association between surgical complications and oncologic outcomes after CLM resection. Whether the link is from indirect factors or from direct consequences, the goal should remain the samestaying on track.

ACKNOWLEDGMENT The University of Texas MD Anderson Cancer Center is supported in part by the National Institutes of Health through Cancer Center Support Grant CA016672.

DISCLOSURE None.

\section{REFERENCES}

1. Matsuda A, Matsumoto S, Seya T, et al. Does postoperative complication have a negative impact on long-term outcomes following hepatic resection for colorectal liver metastasis? A meta-analysis. Ann Surg Oncol. doi:10.1245/s10434-013-2972-z.

2. Tzeng CW, Katz MH, Fleming JB, et al. Risk of venous thromboembolism outweighs post-hepatectomy bleeding complications: analysis of 5651 National Surgical Quality Improvement Program patients. HPB (Oxford). 2012;14:506-13.

3. Aloia TA, Fahy BN, Fischer CP, et al. Predicting poor outcome following hepatectomy: analysis of 2313 hepatectomies in the NSQIP database. HPB (Oxford). 2009;11:510-5.

4. Brouquet A, Mortenson MM, Vauthey JN, et al. Surgical strategies for synchronous colorectal liver metastases in 156 consecutive patients: classic, combined or reverse strategy? J Am Coll Surg. 2010;210:934-41.

5. Mentha G, Majno PE, Andres A, Rubbia-Brandt L, Morel P, Roth AD. Neoadjuvant chemotherapy and resection of advanced synchronous liver metastases before treatment of the colorectal primary. Br J Surg. 2006;93:872-8.

6. Brouquet A, Abdalla EK, Kopetz S, et al. High survival rate after two-stage resection of advanced colorectal liver metastases: response-based selection and complete resection define outcome. J Clin Oncol. 2011;29:1083-90.

7. Adam R, Laurent A, Azoulay D, Castaing D, Bismuth H. Twostage hepatectomy: a planned strategy to treat irresectable liver tumors. Ann Surg. 2000;232:777-85.

8. Kishi Y, Zorzi D, Contreras CM, et al. Extended preoperative chemotherapy does not improve pathologic response and increases postoperative liver insufficiency after hepatic resection for colorectal liver metastases. Ann Surg Oncol. 2010;17:2870-6.

9. Brouquet A, Overman MJ, Kopetz S, et al. Is resection of colorectal liver metastases after a second-line chemotherapy regimen justified? Cancer. 2011;117:4484-92.

10. Reddy SK, Pawlik TM, Zorzi D, et al. Simultaneous resections of colorectal cancer and synchronous liver metastases: a multiinstitutional analysis. Ann Surg Oncol. 2007;14:3481-91.

11. Tzeng CW, Aloia TA, Vauthey JN, et al. Morbidity of staged proctectomy after hepatectomy for colorectal cancer: a matched case-control analysis. Ann Surg Oncol. 2013;20:482-90.

12. Shindoh J, Truty MJ, Aloia TA, et al. Kinetic growth rate after portal vein embolization predicts posthepatectomy outcome: toward zero liver-related mortality in patients with colorectal liver metastases and small future liver remnant. J Am Coll Surg. 2013;216:201-9.

13. Madoff DC, Abdalla EK, Gupta S, et al. Transhepatic ipsilateral right portal vein embolization extended to segment IV: improving hypertrophy and resection outcomes with spherical particles and coils. J Vasc Interv Radiol. 2005; 16:215-25.

14. Kokudo N, Tada K, Seki M, et al. Anatomical major resection versus nonanatomical limited resection for liver metastases from colorectal carcinoma. Am J Surg. 2001;181:153-9. 
15. Figueras J, Burdio F, Ramos E, et al. Effect of subcentimeter nonpositive resection margin on hepatic recurrence in patients undergoing hepatectomy for colorectal liver metastases. Evidences from 663 liver resections. Ann Oncol. 2007;18:1190-5.

16. Chun YS, Vauthey JN, Boonsirikamchai P, et al. Association of computed tomography morphologic criteria with pathologic response and survival in patients treated with bevacizumab for colorectal liver metastases. JAMA. 2009;302:2338-44.
17. Shindoh J, Loyer EM, Kopetz S, et al. Optimal morphologic response to preoperative chemotherapy: an alternate outcome end point before resection of hepatic colorectal metastases. J Clin Oncol. 2012;30:4566-72.

18. Andreou A, Aloia TA, Brouquet A, et al. Margin status remains an important determinant of survival after surgical resection of colorectal liver metastases in the era of modern chemotherapy. Ann Surg. 2012;256(4):642-50. 